LETTER TO THE EDITOR

\title{
NON INVASIVE BRAIN STIMULATION TECHNIQUE - AN AREA NEEDS TO BE EXPLORED
}

Aspiration to enhance human capabilities by increasing cognitive functions and physical performance is a novel approach; however, mechanism to increase human performance to the highest level has been the topic of interest for the researchers since centuries. The activity of human brain can be altered through electrical stimulation that is purely noninvasive. Recently, Studies conducted by rehablitationists have emphasized the use of non-invasive electrical stimulation; one of the non-invasive brain stimulation (NIBS) techniques that induces excitability in human motor cortex is transcranial direct current stimulation (tDCS) that work by process of neuro modulation'. The advantages of NIBS are significant, as it has very rare or no adverse effects and does not require any kind of surgical procedure ${ }^{2}$. On the other hand, there are significant limitations in administering pharmacological treatments, causing non-specific effect and moderate to severe hostile effects ${ }^{3}$. While other contemporary physical and behavioral therapies are much safer and specific, depending on the available resources for therapist and training protocol and patient's cooperation. There is therefore an evident unmet clinical need for the development of new therapeutic approaches in physical and rehabilitation medicine. NIBS is a new approach with positive clinical results explored in the last decade, these methods of brain stimulation are painless and can easily excite and modulate the brain functions. At present, various noninvasive electrical brain stimulation methods have been introduced by commercial providers and now been used by elite sports coaches, that are helping in improving the mental abilities, cognitive functions, balance and also assisting in dealing with the stress ${ }^{4}$. In general, neuromodulation of the human brain can be achieved noninvasively by delivering magnetic, electric and auditory and visual bursts of pulses ${ }^{5}$ to predefined cortical regions. So far, there is little empirical evidence for the efficacy of brain-stimulation techniques on enhancement of sports-specific performance ${ }^{6}$. Hence, as per my understanding and knowledge the research work conducted on such approaches is scarce in Pakistan. Dear Editor, by the virtue of this prestigious journal I would like to draw your kind attention towards this unexplored area of research work, it seems plausible that these emerging techniques will be a future, legitimate way to enhance physical and mental performance.

\section{Muhammad Usman Khan}

Assistant Professor/HOD Physical Education, Health and Sports Sciences,

Ziauddin College of Rehabilitation Sciences

Ziauddin University

\section{REFERENCES}

[1] Jacobson L, Koslowsky M, Lavidor M. tDCS polarity effects in motor and cognitive domains: a meta-analytical review. Experimental brain research. 2012;216(1):1-0.

[2] Bystritsky A, Khalsa SS, Cameron ME, Schiffman J. Current diagnosis and treatment of anxiety disorders. Pharmacy and Therapeutics. 2013;38(1):30.

[3] Finkenzeller T, Würth S, Amesberger G. Consumer-Grade Brain Stimulation Devices in Sports: A Challenge for Traditional Sport Psychology?.J. Appl. Sport Psychol. 2018;30(4):473-93.

[4] Brunoni AR, Vanderhasselt MA. Working memory improvement with non-invasive brain stimulation of the dorsolateral prefrontal cortex: a systematic review and meta-analysis. Brain and cognition. 2014;86:1-9. [5] Tang HY, Riegel B, McCurry SM, Vitiello MV. Open-loop audio-visual stimulation (AVS): A useful tool for management of insomnia?. Applied psychophysiology and biofeedback. 2016;41 (1):39-46.

[6] Angius L, Hopker J, Mauger AR. The ergogenic effects of transcranial direct current stimulation on exercise performance. Frontiers in physiology. 2017:8:90. 